\title{
Template-Based Proxy Caching for Table-Valued Functions
}

\author{
Qiong Luo and Wenwei Xue \\ Department of Computer Science \\ Hong Kong University of Science and Technology \\ Clear Water Bay, Kowloon \\ Hong Kong, China \\ \{luo, wwxue\}@ cs.ust.hk
}

\begin{abstract}
Certain types of database-backed web sites heavily utilize userdefined functions in SQL queries. Unfortunately, existing web proxy caching schemes can not handle these functions. In order to enable proxy caching for such web sites, we propose a template-based proxy caching framework, a function proxy, for table-valued functions. In our framework, function templates are registered with the proxy so that the proxy can answer new queries based on previously cached data in collaboration with the original web site. We identify a common class of function-embedded queries and present several active caching schemes for them. Our experiments with real web sites show the feasibility and usefulness of the function proxy. Additionally, we find that cache-intersecting queries may not be worth handling at the proxy and that containment-based active caching is efficient and practical.
\end{abstract}

\section{Introduction}

Traditionally, user-defined functions are employed in database applications that handle complex data, such as Geographical Information Systems and Computer Aided Design systems. With the proliferating use of the Web, certain types of databasebacked web sites also heavily utilize user-defined functions in answering user queries. While recent research ([1], [13], [14]) has shown the usefulness of advanced web proxy caching for general database-backed web sites, their techniques are not directly applicable to web sites with function-embedded queries, which are queries with embedded calls to use-defined functions. Our goal in this paper is to explore proxycaching techniques for these function-embedded queries.

Function-embedded queries make their presence in applications for functionality extension, performance improvement, and user convenience. Take for example the nearest-neighbor query. First, application-specific distance functions are likely to fall out of the range of DBMS built-in functions. In such case, users must code their own functions and register the functions with the DBMS. Second, users can write their distance functions in a high-level programming language of their choice and employ optimization techniques based on their domain knowledge. As a result, the compiled 
and registered user-defined functions can run efficiently in queries. Last, other users can call the registered functions without knowing the implementation details of the functions, which is especially convenient for non-expert users.

The encapsulation feature of user-defined functions is convenient, but it poses challenges to a proxy that attempts to cache function-embedded queries. Specifically, without knowing the semantics of the user-defined functions, the proxy can only perform the traditional exact-match passive caching. Furthermore, even if a web site is willing to expose the text of its function-embedded queries as well as the code of its functions, the complexity of the functions and the platform-dependency of the implementations may force the proxy to resort to partial or full replication (and porting, if necessary) of the web site. For instance, the SkyServer web site [20] is mirrored in several countries.

Replication and mirroring is a viable solution for database-backed web sites with function-embedded queries. However, it requires significant amounts of resources for deployment and maintenance. Alternatively, we study if we can extend a proxy to perform active caching for function-embedded queries, in which the proxy not only services a function-embedded query that is an exact match to a previous one, but also services a query that can be answered by processing the results of previous queries. We term such a proxy a function proxy.

Active caching in a function proxy requires some semantic information about the functions and the function-embedded queries. We propose to use function templates to specify the semantics of user-defined functions as well as function-embedded query templates to define the types of queries that the proxy answers. These templates are a natural abstraction of the HTML forms for accepting user queries at the web site, the registered user-defined functions with parameters, and the parameterized queries programmed in the web site applications. They enable the proxy to decide on whether and how to cache the query results and to answer new queries by processing the previous results. They also allow the proxy to optimize its cache organization and its limited query processing logic accordingly.

The query processing logic in a function proxy depends on the properties of the templates as well as the caching scheme. More specifically, the proxy needs semantic information of the templates to determine the method to check query containment and overlapping relationships between a new query and a cached query. It needs the same information to answer a cache-contained query from the cache, and it needs further collaboration from the original web site to answer a cache-intersecting query.

We focus our study on table-valued functions (which return a set of tuples) as opposed to scalar functions (which return a scalar value), because the special properties of the former bring additional challenges as well as opportunities for active caching in our function proxy. For instance, compared with a single value returned by a scalar function, the multiple result tuples of a table-valued function consumes more network resource for shipping, but accumulates more data for future query answering.

In addition to designing our function proxy and active caching schemes, we study the issues of proxy caching for user-defined functions in real systems. This is because user-defined functions have application-specific semantics, and caching for such 
workloads is more meaningful in real world applications than in synthetic benchmarks. Fortunately, we find the SkyServer web site [20] for our study.

The SkyServer web site serves terabytes of the public Sloan Digital Sky Survey (SDSS) data for both astronomers and science education. The web site contains many pre-defined functions, such as fGetNearbyObjEq ( $\mathrm{ra}$, dec, radius), fGetObjFromRect (min_ra, max_ra, min_dec, max_dec), and fPhotoFlags (FlagName). These functions are frequently called to serve queries submitted through HTML search forms or invoked from dynamic ASPs. In addition, web users can input arbitrary functionembedded queries directly by using the "SQL" section of the "Search" facility at the web site. The semantic information of the functions as well as the text of the function-embedded queries is available through various online documentations of the web site.

We use the SkyServer as an example to define the templates as well as to describe our function proxy framework in Section 2. We present the template-based active caching scheme implementation for a common class of function-embedded queries in Section 3. We discuss our experimental results with the SkyServer in Section 4. We present related work in Section 5 and draw conclusions in Section 6.

\section{The Template-Based Function Proxy Framework}

As in the recent work on template-based caching ([1], [13]), our key observation in this work is that query templates enable a variety of useful query caching techniques beyond exact matches. There are two reasons for this. One reason is that templates provide the SQL semantics of the queries encoded in HTTP requests, which is essential for enabling web proxies to perform any query processing. The other reason is that templates greatly simplify the required query processing capability at the cache.

Unlike the recent work on template-based caching ([1], [13]), we consider queries that have embedded function calls. Although the queries of the same template are the same up to constant substitution, the embedded functions increase the uncertainty about what query processing (and function execution) capabilities are required at the cache. Therefore, we propose a template-based proxy framework for caching function-embedded queries. This framework facilitates the usage of not only query templates but also function templates in order to enable active caching schemes for function-embedded queries.

Let us use the Radial search form (Figure 1) of the SkyServer as an example to illustrate our proxy framework. This form is used to search the celestial sky around a given point and returns objects (tuples) within a given radius. It has a corresponding function-embedded query template calling a table-valued function named fGetNearbyObjEq(). The corresponding query template of the form is shown in Figure 2.

As can be seen in Figure 2, the table-valued function fGetNearbyObjEq() is called in the FROM clause of the SQL statement. Moreover, the parameters of the function correspond to the user input parameters in the search form. The query template is a join between the result table of the function and the PhotoPrimary table. From the 
documentations of the SkyServer, we know that the join with the PhotoPrimary table is for the purpose of tuple filtering and attribute list expansion.

In addition to the join condition, there are other optional predicates (such as range predicates on $\mathrm{u}, \mathrm{g}, \mathrm{r}$, etc) in the WHERE clause. We use "other_predicates" to represent them, since they are not our focus in this work and they do not affect the properties of the function-embedded queries we study. Finally, there is an optional top-N operation on the tuples of projected attributes.

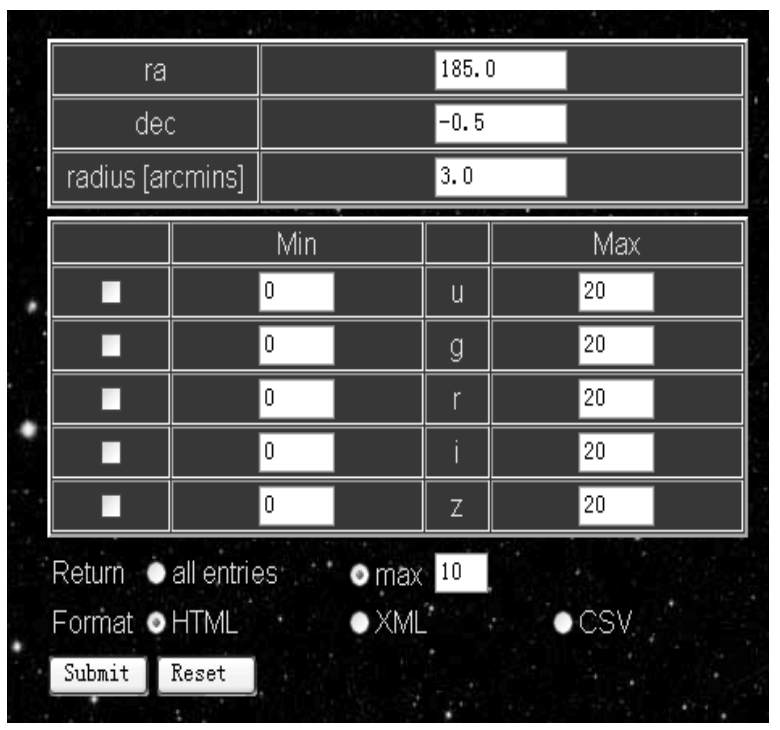

Fig. 1. The Radial search form

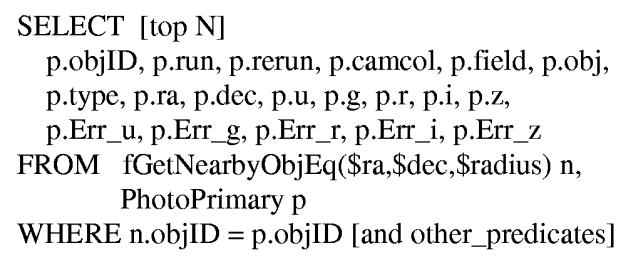

Fig. 2. The function-embedded query template of the Radial search form

Having described function-embedded query templates, let us examine closely the templates for the embedded functions. In our implementation, a function template is an XML file specifying high-level query semantics about the user-defined function. As shown in Figure 3, the function template for the function fGetNearbyObjEq() called in the Radial search form is abstracted as finding all points that are bounded by a 3-D hypersphere. This function template may apply to other functions if they have the same query semantics, even though the meanings of the applications are different. 
In addition to these two templates, we use information files to associate an HTML search form with a function-embedded query template.

Having in place function templates, function-embedded query templates, and the information files for the function-embedded query templates, we illustrate the system architecture of our proxy framework in Figure 4.

\section{Template-Based Active Caching for Function-Embedded Queries}

In this section we first describe the types of function-embedded queries handled, and then present our active caching schemes for the function-embedded queries.

\subsection{Function-Embedded Queries Handled}

Unlike SQL statements, the semantics of user-defined functions can vary widely. This diversity of user-defined functions increases the difficulty of active caching for function-embedded queries. As a first step in applying our proxy caching framework, we identify a common class of function-embedded queries and handle them at the proxy. We summarize the four properties of these queries as follows.

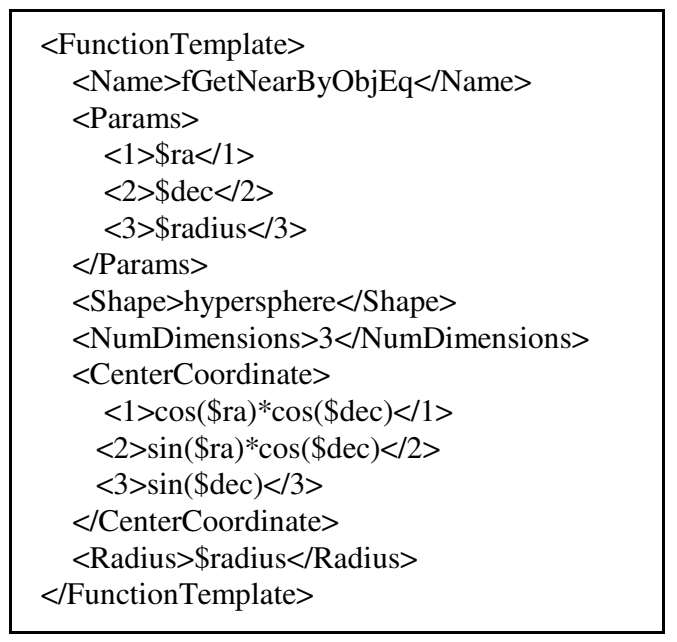

Fig. 3. The function template of function fGetNearbyObjEq()

(1) Determinism. All functions in a DBMS, including user-defined ones, can be split into two groups - deterministic and non-deterministic. Given a fixed database and fixed parameter values of a function (nothing changes over time), the function is deterministic if it always returns the same value each time it is called; otherwise, it 


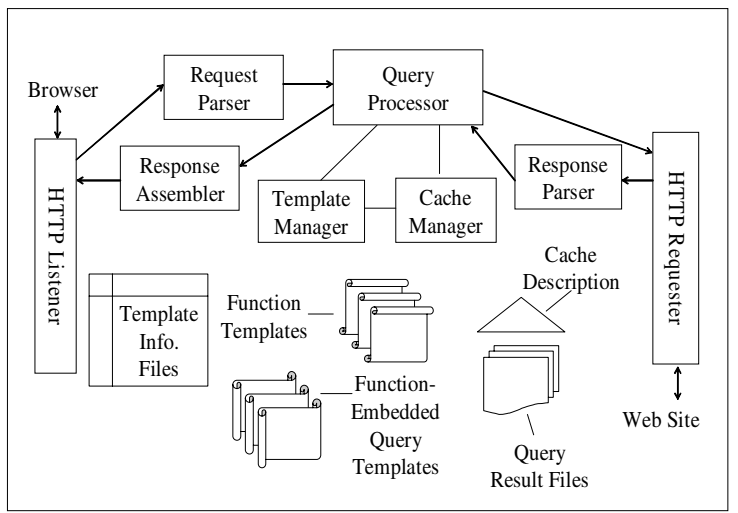

Fig. 4. System architecture

is non-deterministic. By this definition, functions such as getTimeOfDay() is nondeterministic and is not handled by our active caching.

(2) Spatial Region Selection Query Semantics. The tuples in the returned table of an embedded table-valued function are abstracted as points in a multidimensional region. The function is equivalent to a spatial region selection query, which returns all points in the multi-dimensional region. The shape of the region can be a hypercube (most common), a hypersphere, or even a polytope [15] (more complex). As examples, the fGetNearbyObjEq() function of the SkyServer returns all objects within a 3D sphere, and the fGetObjFromRect() function returns all objects within a 2-D rectangular region. Certainly, the functions are not necessarily restricted to spatial applications. For instance, a function of returning books that are similar to a given book, with a certain similarity distance metric over several parameters, can be abstracted into a hypersphere selection query.

(3) Semantics-Preserving Join. If the embedded table-valued function is joined with other tables in the function embedded query, such joins should preserve the query semantics of the table-valued function. For instance, a query from the Radial search form preserves the query semantics of the fGetNearbyObjEq() function, and returns objects within a 3-D sphere with some additional predicates.

(4) Result Attribute Availability. Some attributes are required for checking the spatial relationship between a new query and previously cached queries as well as for evaluating a new query result over previously cached ones. An important case is the attributes that serve as the Cartesian coordinates of the point that a result tuple represents. Therefore, we require that the attributes involved in the query relationship checking and local query evaluation are present in the cached result tuples.

If a function-embedded query has the above four properties, we can transform the problem of checking the relationship between two queries (query exact match, containment, overlapping, or disjoint) into that of checking the spatial relationship between the two corresponding regions. This is the basic idea that we use to implement our active caching schemes for function-embedded queries. 


\subsection{Active Caching Schemes for Function-Embedded Queries}

We have implemented three active caching schemes in our function proxy to investigate the performance tradeoffs among them: (1) the full semantic caching [6], (2) a variant of the full semantic caching that only checks exact match, query containment, and region containment [4], and (3) the containment-based active caching that only checks query containment (including exact matches).

Exact match, query containment, and region containment are all cases of query relationships. Given a new query and the previously cached queries, the proxy uses a spatial relationship checking algorithm on them (without looking at the query results) and returns a status for this new query. The status can be one of the four cases: (a) a new query is an exact match to a cached query, (b) a new query is contained in (or subsumed by) a cached query, (c) a new query overlaps with some cached query (queries), and (d) a new query is disjoint from any cached queries. Region containment is a special case of query overlapping, where the new query contains a region of the cache (one or more cached queries). After the status is identified, the proxy can then evaluate the new query at the proxy correspondingly.

The handling of the two extreme cases (cases a and d) is simple. On one extreme, if the new query is an exact match to a cached query, the proxy will read the cached result and return it to the user. On the other extreme, if the new query is disjoint from all of the cached queries, the proxy will forward the query to and get a response from the original web site, cache this result, and return it to the user.

If the new query is contained in a cached query (case b), the proxy evaluates the new query over the result of that cached query locally and returns the new result to the user. The result of the new query is not cached, since it is already contained in the result of the cached query.

Note that the local evaluation procedure of a subsumed query is determined by the spatial semantics (e.g., the shape and dimensions of region) of the function template and the function-embedded query template. As long as the abstraction of the semantics of an application is correct, the local evaluation procedure will produce correct results for the application. Moreover, this local evaluation implementation is much simpler than the evaluation procedure at the original web site, since the original web site has to deal with a large amount of base data and executes its own (probably more application-specific) implementation of functions. In comparison, the proxy evaluates the new query by selecting the cached result tuples that represent points falling into the multi-dimensional region of the new query. In essence, the evaluation of a subsumed query becomes that of a spatial region selection query over cached results.

The last case is query overlapping (case c), in which the cache can serve only a portion but not all of the answers to the new query. Consequently, the proxy must contact the original web site. The major decision here is whether the proxy sends the original query or a remainder query [6] to the original web site. if the original web site does not support modified queries, i.e., it does not have a remainder query facility, the proxy has no choice but always sends the original query to the web site. If the web site has a facility to handle remainder queries, we further consider the tradeoffs in designing the active caching schemes. 
The main tradeoff is among the processing cost at the proxy, the processing cost at the server, and the transfer cost on the network. On one hand, sending a remainder query can improve cache utilization (providing some answers from the cache) and reduce the network bandwidth consumption, since ideally fewer result tuples are returned from the web site. On the other hand, it may not reduce the query processing time at the web site since a remainder query is usually more complicated than the original query, and sometimes the time for evaluating the probing query [6] and merging the two parts of the results at the cache can be significant.

Finally, in the case of region containment (a special case in query overlapping), our proxy merges the results of all of the cached queries with the result of the remainder query to form the final result of the new query. After answering this new query, the result of the new query is cached and the cached results of all those subsumed queries are removed. This special case deserves attention, because it reduces the number of cached queries and improves cache utilization.

\section{Experimental Evaluation}

In this section, we examine the feasibility of our function proxy and the performance of active caching schemes using real query traces extracted from the SkyServer web logs. We focus our experiments on caching the query results of the Radial web search form at the SkyServer, and show only a few results for space consideration.

\subsection{Experimental Environment}

We used two computers in our experiments. Both machines have a Pentium IV 1.8 $\mathrm{GHz}$ CPU and are running the Windows XP Professional operating system. The machine for running an RBE (Remote Browser Emulator, the program we write for emulating a web browser client) has $256 \mathrm{MB}$ main memory and 60GB disk space. The machine that runs as the function proxy has $1 \mathrm{~GB}$ main memory and 100GB disk space.

We implemented our function proxy as a Java servlet on top of the Apache Tomcat servlet engine version 4.1.24 [2]. We implemented the three active caching schemes for function-embedded queries within the function proxy framework. We also implemented the passive caching (handling exact matches only) in the proxy for comparison purposes. We registered the query templates and functions templates of the Radial web search form of the SkyServer with our proxy. We ran the Radial form query trace through our proxy with various configurations to the SkyServer web site.

The query trace for the Radial search form has a total of 11,323 queries. With an unlimited cache size, nearly 51\% (17\% query exact match and $34 \%$ query containment) of the Radial search form queries can be completely answered by the cache. Additionally, about $9 \%$ of the queries overlap.

Since the SkyServer allows web users to submit arbitrary SQL queries (http:// skyserver.sdss.org/en/tools/ search/sql.asp), we used this interface as the remainder query facility in our experiments. 


\subsection{Experimental Results}

We ran the Radial query trace through our function proxy in various configurations to the SkyServer. A configuration includes the caching scheme, the cache description implementation, and the cache size. The caching scheme can be no cache, a passive cache, or an active cache. An active cache can be one of the three alternatives we implemented - (1) the full semantic caching, (2) the variant of full semantic caching handling exact match, query containment, and region containment, and (3) the containment-based active caching. The cache description implementation can be an array or an R-tree.

The two performance metrics used in our study are response time and cache efficiency. The response time of a query is measured at the browser emulator. The cache efficiency of a query is defined as the percentage of the result tuples that are served from the proxy cache to the total number of result tuples of the query. The average cache efficiency of a query trace is the arithmetic average of the cache efficiency values of all queries. It reveals the cache utilization more accurately than a cache hit ratio. Additionally, the proxy servlet records timing information in each step of query processing for the purpose of a detailed analysis.

We first examined the performance impact of an active caching scheme in comparison with a non-caching (tunneling) proxy (NC) and a proxy with passive caching (PC). We picked the full semantic caching as the active caching scheme since it exercises all aspects of active caching. We further compared the performance impact of the cache description implementation of the active caching - ACR is the active caching with an R-tree cache description and ACNR is the active caching with an array cache description. In addition, the cache size was varied from $1 / 6$ of the total result size of the query trace to the full result size (nearly $300 \mathrm{MB}$ XML files).

Figure 5 illustrates the average response time of the first 10,000 queries of the trace under various proxy configurations. Table 1 summarizes the average cache efficiency of active caching (which is the same for ACNR and ACR) and passive caching on the trace.

Table 1 shows that the cache efficiency of passive caching is about $30 \%$ and that of active caching is 53-59\%. Apparently, the cache efficiency of active caching is much higher than that of passive caching. Moreover, the increase in cache size improves cache efficiency better for active caching than for passive caching.

Associating Table 1 with Figure 5, we see that the general trend in response time corresponds to that in cache efficiency. Without any caching (NC), the average response time is more than 2 seconds. With passive caching, it goes down to around 1.4 seconds (30\% improvement over NC). With active caching, it goes down further to around 1.2 seconds. However, the average response time of either caching scheme

Table 1. Average cache efficiency of AC and PC

\begin{tabular}{|c|c|c|c|c|}
\hline Cache Size & $1 / 6$ & $1 / 3$ & $1 / 2$ & 1 \\
\hline AC & 0.531 & 0.565 & 0.582 & 0.593 \\
\hline PC & 0.290 & 0.305 & 0.311 & 0.313 \\
\hline
\end{tabular}




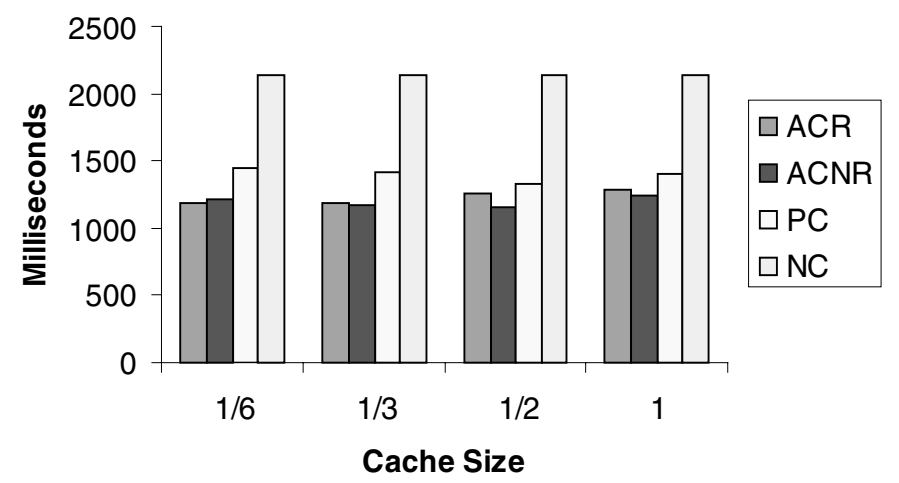

Fig. 5. Average response time

does not improve much when the cache size increases. his is because response time is not only affected by cache efficiency, but also by cache maintenance cost.

Interestingly, Figure 5 shows that the R-tree index on the cache description does not accelerate the active caching scheme and in some cases even slows it down slightly. The main reason is that the size of the cache description is small so that a linear search and a tree search have similar main memory performance. A more detailed study on the time breakdown of individual queries revealed that the cache checking time with or without the R-tree index is always under 100 milliseconds. Finally, the maintenance of the R-tree index is more costly than that of an array.

We find that cache-intersecting queries are not always helpful for active caching (results omitted). Therefore, we compared the full semantic caching with the other two active caching alternatives which have less or no handling of cache-intersecting queries. In Figure 6, the "First" is full semantic aching, the "Second" is active caching without handling query overlapping other than region containment, and the "Third" is pure query containment based active caching. The results shown in Figure 6 were for an unlimited cache size and with an array-based cache description. The latter two schemes have slightly worse cache efficiency than that of the first $(0.544$ and 0.511 respectively as opposed to 0.593 ), but they outperform the full semantic caching in response time. This highlights the usefulness of containment-based active caching, which does not require a high degree of cooperation with the web sites.

\section{Related Work}

Semantic caching [6] and predicate caching [10] was initially proposed in traditional client-server database architectures. Such semantic caching has been extended for keyword queries in a web meta-searcher for heterogeneous web sources [4], and further applied to web proxies for database-backed web sites ([1], [13]). As one variant of our active caching schemes, full semantic caching is also studied in our function proxy for table-valued functions. 


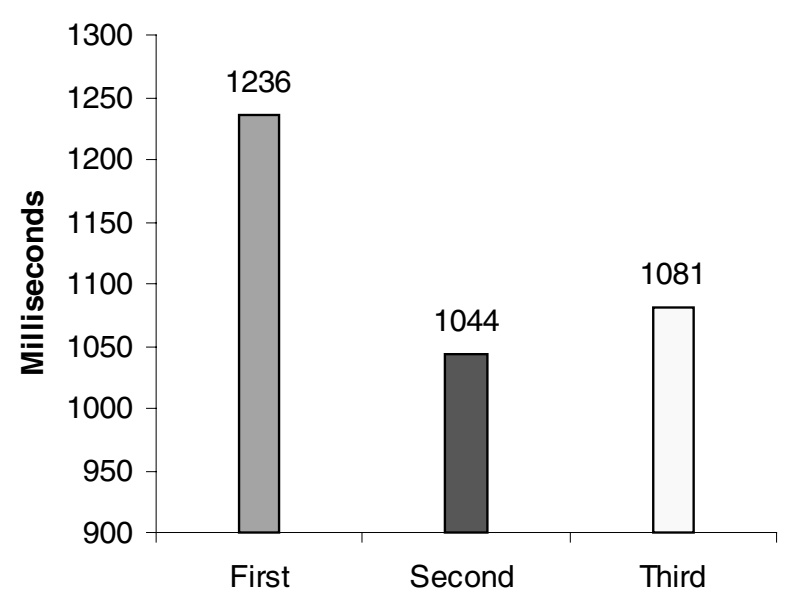

Fig. 6. Average response time of active caching schemes

User-defined functions are widely supported in commercial DBMS products ([9], [16]). The SkyServer ([20]) is a representative real world application of these functions. Hellerstein and Naughton proposed a query execution technique called Hybrid Cache [8] for caching the results of expensive methods in a full-fledge DBMS. The MOCHA system [19] implemented a migration paradigm for shipping applicationspecific Java code around distributed data sources. The Active Cache interface [3] allows web servers to send cache applets to web proxies. In comparison, our work focuses on caching for table-valued functions in a web proxy, as opposed to migration or execution of scalar functions.

Our work is also related to answering queries using views ([7], [11], [18], [21]). Although table-valued functions can be regarded as a kind of parameterized views, they usually have non-SQL semantics (as an evidence, they are often implemented in programming languages other than SQL). Consequently, known view selection and view matching algorithms are not directly applicable to table-valued functions. Moreover, our function proxy has only a limited query processing capability such that we chose to answer new function-embedded queries fully at the proxy based on query-containment checking.

Finally, our work complements recent research on various active proxy caching techniques, some of which do not specifically address database queries ([3], [5]), and some address database queries but not function-embedded queries ([1], [12], [13], [14]).

\section{Conclusions}

We have proposed a template-based proxy caching framework for database-backed web sites with queries calling table-valued functions. The function proxy operates 
around function templates and function-embedded query templates, which describe high-level query semantics. We have proposed several active caching schemes for a common class of function-embedded queries that have spatial region selection query semantics. We transform the checking of query containment and query overlap into spatial region relationship checking with no need of examining the query results. We further implement the local evaluation procedure of these queries by exploiting their query semantics. Our experimental results with real web sites and real query traces show the feasibility and usefulness of the function proxy. Additionally, we find that while containment-based active caching is efficient and practical, answering cacheintersecting queries may not be worthwhile.

A full version of this paper is available at http://www.cs.ust.hk/ luo.

Acknowledgements. We would like to thank Jim Gray at Microsoft Bay Area Research Center for making the personal SkyServer code and data available as well as answering our questions about the SkyServer. This research was supported by Grant HKUST6158/03E from Hong Kong Research Grant Council.

\section{References}

1. Khalil Amiri, Sanghyun Park, Renu Tewari, and Sriram Padmanabhan. Scalable TemplateBased Query Containment Checking for Web Semantic Caches. ICDE 2003.

2. The Apache Tomcat Servlet Engine. http://jakarta.apache.org/tomcat/index.html.

3. Pei Cao, Jin Zhang, and Kevin Beach. Active Cache: Caching Dynamic Contents on the Web. Middleware 1998.

4. Boris Chidlovskii, Claudia Roncancio, and Marie-Luise Schneider. Semantic Cache Mechanism for Heterogeneous Web Querying. WWW8, 1999.

5. Anindya Datta, Kaushik Dutta, Helen Thomas, Debra Vandermeer, Suresha, and Krithi Ramamritham. Proxy-Based Acceleration of Dynamically Generated Content on the World Wide Web: An Approach and Implementation. SIGMOD 2002.

6. Shaul Dar, Michael J. Franklin, Björn T. Jónsson, Divesh Srivastava, and Michael Tan. Semantic Data Caching and Replacement. VLDB 1996.

7. Jonathan Goldstein and Per-Åke Larson. Optimizing Queries Using Materialized Views: A Practical, Scalable Solution. SIGMOD 2001.

8. Joseph M. Hellerstein and Jeffrey F. Naughton. Query Execution Techniques for Caching Expensive Methods. SIGMOD 1996.

9. IBM DB2. http://www-3.ibm.com/software/data/db2/udb/

10. Arthur M. Keller and Julie Basu. A Predicated-based Caching Scheme for Client-Server Database Architectures. VLDB Journal 5(1): 35-47 (1996).

11. Alexandros Labrinidis and Nick Roussopoulos. WebView Materialization. SIGMOD2000.

12. Thanasis Loukopoulos, Panos Kalnis, Ishfaq Ahmad, and Dimitris Papadias. Active Caching of On-Line-Analytical-Processing Queries in WWW Proxies. ICPP 2001: 419426.

13. Qiong Luo and Jeffery F.Naughton. Form-Based Proxy Caching for Database-Backed Web Sites. VLDB 2001: 191-200.

14. Qiong Luo, Jeffrey F. Naughton, Rajasekar Krishnamurthy, Pei Cao, and Yunrui Li. Active Query Caching for Database Web Servers. WebDB 2000. 
15. MathWorld. http://mathworld.wolfram.com/topics/MultidimensionalGeometry.html.

16. MSDN Library: Working with Stored Procedures and User-Defined Functions. http://msdn.microsoft.com/library/default.asp

17. Personal SkyServer. http://research.microsoft.com/ gray/SDSS/personal_skyserver.htm.

18. Rachel Pottinger and Alon Halevy. A Scalable Algorithm for Answering Queries Using Views. VLDB 2000.

19. Manuel Rodriguez-Martinez and Nick Roussopoulos. MOCHA: A Self-Extensible Database Middleware System for Distributed Data Sources. SIGMOD 2000.

20. The SkyServer web site. http://skyserver.sdss.org/

21. Divesh Srivastava, Shaul Dar, H. V. Jagadish, and Alon Y. Levy. Answering Queries with Aggregations Using Views. VLDB 1996. 